\title{
ON ORTHOGONALLY SCATTERED DILATIONS OF BOUNDED VECTOR MEASURES
}

\author{
HANNU NIEMI
}

1. Introduction. Let $H$ be a Hilbert space with complex scalars and let $S$ be a locally compact Hausdorff space. In this paper we show that every bounded $H$-valued vector measure $\mu$ defined on $S$ is, in a certain sense, a projection of a bounded orthogonally scattered vector measure $\mu^{\prime}$ defined on $S$ with values in a larger Hilbert space $H^{\prime}$.

Our result is a generalization of Abreu's Theorem 3.1 in [2] (see also the proof of the main theorem in Abreu's paper [1]), where he presented a sufficient condition for a bounded $H$-valued vector measure to be a "projection" of a bounded orthogonally scattered vector measure with values in a larger Hilbert space $H^{\prime}$.

The proof of our result is essentially based on a factorization theorem of Grothendieck [5; p. 52] and on a construction used by Abreu in [1] and [2].

A detailed proof of Grothendieck's theorem (in another form) was presented by Rogge [11] in the case of bounded vector measures defined on a compact Hausdorff space and taking values in a real Hilbert space. Rogge's work was based on some earlier results of Pietsch [10]. For the sake of completeness we show in Section 2 that these results of Rogge and Pietsch are applicable even in the case considered by us.

2. On 2-majorizable vector measures with values in a Hilbert space. In this section we show that the results of Rogge [11] and Pietsch [10] concerning the socalled 2-majorizable vector measures are valid even for vector measures, which are defined on a locally compact Hausdorff space and taking values in a complex Hilbert space.

Let $T$ be a compact Hausdorff space. By $C(T)$ we denote the linear space of all continuous functions ${ }^{1} f: T \rightarrow C$. Similarly, for a locally compact Hausdorff space $S$ we denote by $C_{0}(S)$ the linear space of all continuous functions $f: S \rightarrow C$

${ }^{1}$ In this paper we denote by $N, R$ and respectively by $C$ the set of natural, real and respectively complex numbers.

doi:10.5186/aasfm. 1977.0320 
vanishing at infinity, i.e., a continuous function $f: S \rightarrow C$ is an element of $C_{0}(S)$ if for every $\varepsilon>0$ there exists a compact set $K \subset S$ such that $|f(s)|<\varepsilon$ for all $s \notin K$. The linear subspace of all real-valued functions in $C(T)$ (resp. in $\left.C_{0}(S)\right)$ is denoted by $C^{\mathrm{Re}}(T)$ (resp. by $C_{0}^{\mathrm{Re}}(S)$ ). The topology of $C(T)$ and $C_{0}(S)$ is defined by the supremum norm.

Next we recall some definitions concerning Radon measures and vector measures. For convenience we present them in the case of a locally compact Hausdorff space and the complex scalars. The definitions in the case of a compact Hausdorff space or in the case of the real scalars are similar.

Let $S$ be a locally compact Hausdorff space. The set of all bounded Radon measures on $S$, i.e., the dual of $C_{0}(S)$ is denoted by $\mathscr{M}_{C}^{1}(S)$. We recall that a Radon measure $v \in \mathscr{M}_{C}^{1}(S)$ is said to be real-valued, if $\overline{v(\bar{f})}=v(f)$ for all $f \in C_{0}(S)$. In this paper we call a real-valued $v \in \mathscr{M}_{C}^{1}(S)$ positive, if $v(f) \geqq 0$ for all $f \in C_{0}(S)$, $f \geqq 0$.

Let $H$ be a Hilbert space. By (.|.) or by $(. \mid .)_{H}$ we denote the inner product and respectively by $\|\cdot\|$ or by $\|\cdot\|_{H}$ the norm of $H$. The topology of a Hilbert space is always the norm topology.

In this paper we use the integration technique of vector measures introduced by Thomas [12]. We use even Thomas' terminology, i.e., we use the term integrable when Bourbaki [4] uses the term essentially integrable.

Let $H$ be a Hilbert space with complex scalars and let $S$ be a locally compact Hausdorff space. We recall that a bounded $H$-valued vector measure $\mu$ on $S$ is a continuous linear mapping $\mu: C_{0}(S) \rightarrow H$. For a bounded $H$-valued vector measure $\mu$ on $S$ (or for a Radon measure $\mu \in \mathscr{M}_{C}^{1}(S)$ ) the linear space of functions $f: S \rightarrow C$ for which the function $|f|^{p}$ is $\mu$-integrable is denoted by $\mathscr{L}_{C}^{p}(\mu), \quad p \geqq 1$; and the integral of a function $f \in \mathscr{L}_{C}^{1}(\mu)$ with respect to $\mu$ is denoted by

$$
\int f d \mu \text {. }
$$

Moreover, we denote by $\overline{\mathrm{sp}}\{\mu\}$ the closed linear subspace in $H$ spanned by the set $\left\{\mu(f) \mid f \in C_{0}(S)\right\}$. It follows from the completeness of $\overline{\mathrm{sp}}\{\mu\}$ and from the way to define the integral of a vector measure (see Thomas [12; pp. 65-69]) that

$$
\int f d \mu \in \overline{\mathrm{sp}}\{\mu\} \quad \text { for all } f \in \mathscr{L}_{C}^{1}(\mu) \text {. }
$$

The norm of a bounded $H$-valued vector measure $\mu$ on $S$, i.e., the norm of the continuous linear mapping $\mu: C_{0}(S) \rightarrow H$ is denoted by $\|\mu\|$.

The definition of a 2-majorizable (or more generally $p$-majorizable; $1 \leqq p<\infty$ ) vector measure is essentially due to Persson and Pietsch (see Pietsch [10] and references given there).

Definition 1. Let $H$ be a Hilbert space with complex (resp. real) scalars and let $S$ be a locally compact Hausdorff space. A bounded vector measure $\mu: C_{0}(S) \rightarrow H$ (resp. $\mu: C_{0}^{\mathrm{Re}}(S) \rightarrow H$ ) is called 2-majorizable, if there exists a bounded positive 
Radon measure $v: C_{0}(S) \rightarrow C$ (resp. $v: C_{0}^{\mathrm{Re}}(S) \rightarrow R$ ) such that

$$
\left.\|\mu(f)\|_{H} \leqq v\left(|f|^{2}\right)^{1 / 2} \quad \text { for all } f \in C_{0}(S) \quad \text { (resp. } f \in C_{0}^{\mathrm{Re}}(S)\right) .
$$

For a bounded 2-majorizable $H$-valued vector measure $\mu: C_{0}(S) \rightarrow H \quad$ (resp. $\mu: C_{0}^{\operatorname{Re}}(S)$ $\rightarrow H$ ) the set of all bounded positive Radon measures $v: C_{0}(S) \rightarrow C$ (resp. $v: C_{0}^{\mathrm{Re}}(S)$ $\rightarrow R$ ) satisfying the condition (1) is denoted by $\mathscr{M}_{\mu}^{2}$. The elements of $\mathscr{M}_{\mu}^{2}$ are called 2-majorants of $\mu$.

The following lemma is essentially due to Thomas [12; p. 98]. In the proof of the lemma we use the semi-variation $\mu \bullet$ of a vector measure $\mu$. The semi-variation of a vector measure is defined by Thomas [12; pp. 65-66].

Lemma 2. Let $S$ be a locally compact Hausdorff space and let $T=S \cup\{\infty\}$ be an Alexandroff compactification of $S$. If $H$ is a real Hilbert space, then for every bounded vector measure $\mu: C_{0}^{\mathrm{Re}}(S) \rightarrow H$ there exists a bounded vector measure $\tilde{\mu}: C^{\operatorname{Re}}(T) \rightarrow H$ such that $\|\tilde{\mu}\|=\|\mu\|$ and

$$
\mu(f)=\tilde{\mu}(\tilde{f}) \quad \text { for all } f \in C^{\mathrm{Re}}(S)
$$

here $\tilde{f}: T \rightarrow R$ is defined by $\tilde{f}(s)=f(s), s \in S$, and $\tilde{f}(\infty)=0$.

Proof. Let $\mu: C_{0}^{\mathrm{Re}}(S) \rightarrow H$ be a bounded vector measure. Since $\mu$ is a continuous linear mapping from a normed space to a Hilbert space it is weakly compact. Thus all bounded continuous functions $f: S \rightarrow R$ are $\mu$-integrable (Thomas [12; pp. 86-87]).

We define a linear mapping $\tilde{\mu}: C^{\mathrm{Re}}(T) \rightarrow H$ by setting

$$
\tilde{\mu}(f)=\int f_{0} d \mu+f(\infty) \int 1 d \mu, \quad f \in C^{\mathrm{Re}}(T) ;
$$

here $f_{0}$ is the restriction of $f$ to $S$ and 1 stands for the constant function $g(s)=1$ for all $s \in S$. Then $\tilde{\mu}$ is a bounded vector measure on $T$, since using the properties of $\mu \cdot$ (Thomas [12; pp. 68-69]) we get

$$
\begin{aligned}
\|\tilde{\mu}(f)\| & \leqq\left\|\int f_{0} d \mu\right\|+\left\|f(\infty) \int 1 d \mu\right\| \\
& \leqq \mu^{\bullet}\left(\left|f_{0}\right|\right)+|f(\infty)| \mu^{\bullet}(1) \\
& \leqq \mu^{\bullet}(1) \sup |f|, \quad f \in C^{\mathrm{Re}}(T) .
\end{aligned}
$$

Since $\|\mu\|=\mu \cdot(1)$ (Thomas [12; p. 86 and p. 69]), we get $\|\mu\|=\|\tilde{\mu}\|$.

Clearly, $\mu(f)=\tilde{\mu}(\tilde{f})$ for all $f \in C_{0}^{\mathrm{Re}}(S)$, which proves the lemma.

Let $H$ be a Hilbert space with complex scalars. In the following we denote by $H_{\mathrm{Re}}$ the corresponding real Hilbert space.

Suppose $\mu$ is a bounded $H$-valued vector measure defined on a locally compact Hausdorff space $S$. We define a bounded vector measure $\mu_{\mathrm{Re}}: C_{0}^{\mathrm{Re}}(S) \rightarrow H_{\mathrm{Re}}$ by setting

$$
\mu_{\mathrm{Re}}(f)=\mu(f), \quad f \in C_{0}^{\mathrm{Re}}(S)
$$


Lemma 3. Let $H$ be a Hilbert space with complex scalars. Then for all bounded $H$-valued vector measures $\mu$ defined on a locally compact Hausdorff space $S$

$$
\mathscr{M}_{\mu}^{2} \supset\left\{4 \tilde{v} \in \mathscr{M}_{C}^{1}(S) \mid v \in \mathscr{M}_{\mu_{\mathrm{Re}}}^{2}\right\} ;
$$

here $\tilde{v}(f)=v(\operatorname{Re} f)+i v(\operatorname{Im} f), f \in C_{0}(S)$.

Proof. Suppose a bounded positive Radon measure $v: C_{0}^{\mathrm{Re}}(S) \rightarrow R$ is a 2-majorant of $\mu_{\mathrm{Re}}$. Then for all $f \in C_{0}(S)$

$$
\begin{aligned}
\|\mu(f)\|_{H} & \leqq\|\mu(\operatorname{Re} f)\|_{H}+\|\mu(\operatorname{Im} f)\|_{H} \\
& =\left\|\mu_{\operatorname{Re}}(\operatorname{Re} f)\right\|_{H_{\mathrm{Re}}}+\left\|\mu_{\operatorname{Re}}(\operatorname{Im} f)\right\|_{H_{\mathrm{Re}}} \\
& \leqq v\left(|\operatorname{Re} f|^{2}\right)^{1 / 2}+v\left(|\operatorname{Im} f|^{2}\right)^{1 / 2} \leqq 2 v\left(|f|^{2}\right)^{1 / 2} .
\end{aligned}
$$

Thus $4 \tilde{v}$ is a 2 -majorant of $\mu$, which proves the lemma.

Let $H$ be a Hilbert space with complex scalars. It follows from Lemma 2 and Lemma 3 that the results of Rogge [11; Satz 1] and Pietsch [10; Satz 1] concerning 2-majorizable vector measures are applicable for all bounded $H$-valued vector measures defined on a locally compact Hausdorff space. We state these results as a theorem.

Theorem 4. Let $H$ be a Hilbert space with complex scalars and let $\mu$ be a bounded H-valued vector measure defined on a locally compact Hausdorff space S. Then $\mu$ is 2-majorizable.

3. Orthogonally scattered dilations of vector measures with values in a Hilbert space. In this section $S$ stands for a locally compact Hausdorff space and the scalar field of all Hilbert spaces under consideration is $C$.

In this section we use so-called bimeasures. We recall that a bounded bimeasure $B$ on $S \times S$ is defined as a continuous bilinear form $B: C_{0}(S) \times C_{0}(S) \rightarrow C$.

Bimeasures and their integration have been especially studied in the paper of Morse and Transue [7] and in the paper of Thomas [12; pp. 144-147]. We have used bimeasures in analyzing vector measures with values in a Hilbert space in our papers [8] and [9].

Definition 5. Let $H$ be a Hilbert space and let $\mu$ be an H-valued vector measure on $S$. The (continuous) bilinear form

$$
B(f, g)=(\mu(f) \mid \mu(\bar{g})), \quad f, g \in C_{0}(S),
$$

is called the bimeasure defined by $\mu$.

Definition 6. A bounded vector measure $\mu$ on $S$ with values in a Hilbert space $H$ is said to be orthogonally scattered, if

$$
(\mu(f) \mid \mu(g))=0
$$

for all $f, g \in C_{0}(S)$ such that the supports of $f$ and $g$ are compact and disjoint. 
Orthogonally scattered vector measures (with values in a Hilbert space) have been especially studied by Masani [6]. In [9] we gave several characterizations of an orthogonally scattered vector measure using the bimeasure defined by it. In this paper we make use of the characterization stated in the following theorem. For the proof see [9; Theorem 24].

Theorem 7. Let $H$ be a Hilbert space. A bounded H-valued vector measure $\mu$ on $S$ is orthogonally scattered if and only if there exists a positive $v \in \mathscr{M}_{C}^{1}(S)$ such that

$$
(\mu(f) \mid \mu(g))=v(f \bar{g}) \quad \text { for all } f, g \in C_{0}(S) ;
$$

here $(f \bar{g})(s)=f(s) \overline{g(s)}, s \in S$. If there exists a bounded positive Radon measure $v \in \mathscr{M}_{C}^{1}(S)$ satisfying the condition (2), then it is unique and $\mathscr{L}_{C}^{2}(v)=\mathscr{L}_{C}^{1}(\mu)$.

Let $H$ be a Hilbert space and let $M$ be a closed linear subspace of $H$. Then the orthogonal projection of $H$ to $M$ is denoted by $P_{M}$.

Definition 8. Let $H$ be a Hilbert space and let $\mu$ be a bounded $H$-valued vector measure on $S$. A triple $\left(H^{\prime}, \mu^{\prime}, j\right)$ consisting of a Hilbert space $H^{\prime}$, of an $H^{\prime}$-valued bounded vector measure $\mu^{\prime}$ on $S$ and of a linear mapping $j: \overline{s p}\{\mu\} \rightarrow H^{\prime}$ is said to be an orthogonally scattered dilation of the pair $(H, \mu)$, if

(i) $\mu^{\prime}$ is orthogonally scattered,

(ii) $j: \overline{\mathrm{sp}}\{\mu\} \rightarrow j(\overline{\mathrm{sp}}\{\mu\})$ is an isometric isomorphism and if

(iii) $P_{j(\overline{\mathrm{sp}}\{\mu\})} \circ \mu^{\prime}(f)=j \circ \mu(f)$ for all $f \in C_{0}(S)$.

The following theorem is a direct consequence of Theorem 7 and a general result of Thomas [12; pp. 78-79].

Theorem 9. Let $\mu$ be a bounded vector measure on $S$ with values in a Hilbert space $H$ and let $\left(H^{\prime}, \mu^{\prime}, j\right)$ be an orthogonally scattered dilation of $(H, \mu)$. Then $\mathscr{L}_{C}^{1}(\mu)$ $=\mathscr{L}_{C}^{1}(j \circ \mu)$ and

$$
j\left(\int f d \mu\right)=\int f d(j \circ \mu) \quad \text { for all } f \in \mathscr{L}_{C}^{1}(\mu) .
$$

If $v \in \mathscr{M}_{C}^{1}(S)$ is the unique bounded positive Radon measure on $S$ for which

$$
\left(\mu^{\prime}(f) \mid \mu^{\prime}(g)\right)=v(f \bar{g}), \quad f, g \in C_{0}(S) ;
$$

then $\mathscr{L}_{C}^{2}(v)=\mathscr{L}_{C}^{1}\left(\mu^{\prime}\right) \subset \mathscr{L}_{C}^{1}(\mu)$. Furthermore,

$$
P_{j(\overline{\operatorname{sp}}\{\mu\})}\left(\int f d \mu^{\prime}\right)=\int f d(j \circ \mu) \quad \text { for all } f \in \mathscr{L}_{C}^{1}\left(\mu^{\prime}\right) .
$$

Let $\mu$ be a bounded vector measure on $S$ with values in a Hilbert space $H$. We present next a necessary and sufficient condition for the existence of an orthogonally scattered dilation of the pair $(H, \mu)$.

First we present some preliminary results.

Definition 10. A bounded bimeasure $B$ on $S \times S$ is called positive definite, if

$$
B(f, \bar{f}) \geqq 0 \quad \text { for all } \quad f \in C_{0}(S) .
$$


Remark. Let $E$ be an arbitrary set. We recall that a mapping $r: E \times E \rightarrow C$ is said to be positive definite or a positive definite kernel, if

$$
\sum_{j=1}^{m} \sum_{k=1}^{m} a_{j} \bar{a}_{k} r\left(s_{j}, s_{k}\right) \geqq 0
$$

for all $a_{j} \in C, s_{j} \in E, j=1, \ldots, m, m \in N$.

If $B$ is a bounded positive definite bimeasure on $S \times S$, then the mapping $Q: C_{0}(S) \times C_{0}(S) \rightarrow C$,

$$
Q(f, g)=\overline{B(\bar{f}, g)}, \quad f, g \in C_{0}(S),
$$

is a positive definite kernel.

Let $E$ be an arbitrary set and let $r: E \times E \rightarrow C$ be a positive definite kernel. For $t \in E$ we define $r_{t}: E \rightarrow C$ by setting

$$
r_{t}(s)=r(s, t), \quad s \in E .
$$

It is a well-known fact that for the positive definite kernel $r$ there exists a (unique) Hilbert space $H(r)$ consisting of a linear space of functions $f: E \rightarrow C$, such that $r_{t} \in H(r)$ for all $t \in E$;

$$
\left(r_{s} \mid r_{t}\right)_{H(r)}=r(t, s) \quad \text { for all } s, t \in E ;
$$

and the closed linear subspace in $H(r)$ spanned by the set $\left\{r_{t} \mid t \in E\right\}$ coincides with $H(r)$. The space $H(r)$ is called the reproducing kernel Hilbert space spanned by $r$ (Aronszajn [3]).

The following lemma is a direct consequence of the properties of reproducing kernel Hilbert spaces listed above.

Lemma 11. Let $B$ be a bounded positive definite bimeasure on $S \times S$. Define

Then the mapping

$$
Q(g, h)=\overline{B(\bar{g}, h)}, \quad g, h \in C_{0}(S) .
$$

$$
\mu_{Q}(f)=Q_{f}, \quad f \in C_{0}(S),
$$

is a bounded vector measure on $S$ with values in the reproducing kernel Hilbert space $H(Q)$ spanned by $Q$ and

$$
\left(\mu_{Q}(f) \mid \mu_{Q}(g)\right)_{H(Q)}=B(f, \bar{g}), \quad \text { for all } f, g \in C_{0}(S) \text {. }
$$

Let $H$ be a Hilbert space. The following theorem characterizes the bounded $H$-valued vector measures on $S$ for which there exists an orthogonally scattered dilation of $(H, \mu)$. In the proof of the theorem we use the direct sum of two reproducing kernel Hilbert spaces. This idea originates from Abreu's papers [1] and [2].

Theorem 12. Let $\mu$ be a bounded vector measure on $S$ with values in a Hilbert space $H$. Then there exists an orthogonally scattered dilation $\left(H^{\prime}, \mu^{\prime}, j\right)$ of $(H, \mu)$ if and only if there exists a positive $v \in \mathscr{M}_{C}^{1}(S)$ such that

$$
\|\mu(f)\|_{H}^{2} \leqq v\left(|f|^{2}\right) \quad \text { for all } f \in C_{0}(S) .
$$


For every positive $\nu \in \mathscr{M}_{C}^{1}(S)$ satisfying the condition (4) there exists an orthogonally scattered dilation $\left(H^{\prime}, \mu^{\prime}, j\right)$ of $(H, \mu)$ such that

$$
\left(\mu^{\prime}(f) \mid \mu^{\prime}(g)\right)_{H^{\prime}}=v(f \bar{g}) \quad \text { for all } f, g \in C_{0}(S) .
$$

Proof. The necessity of the condition (4) is obvious.

To prove the sufficiency we first note that in the case

$$
\|\mu(f)\|_{H}^{2}=v\left(|f|^{2}\right) \quad \text { for all } f \in C_{0}(S)
$$

the vector measure $\mu$ is by Theorem 7 orthogonally scattered, since applying a wellknown polarization formula we get

$$
(\mu(f) \mid \mu(g))_{H}=v(f \bar{g}) \quad \text { for all } f, g \in C_{0}(S) .
$$

Thus, in this case the triple $(H, \mu, i)$, where $i: H \rightarrow H$ is the identity mapping, is an orthogonally scattered dilation of $(H, \mu)$.

Suppose there exists a function $f_{0} \in C_{0}(S)$ for which

$$
\left\|\mu\left(f_{0}\right)\right\|_{H}^{2}<v\left(\left|f_{0}\right|^{2}\right) .
$$

Let $B$ be the bimeasure defined by $\mu$. We define a bounded bimeasure $B^{\prime} \neq 0$ on $S \times S$ by setting

$$
B^{\prime}(f, g)=v(f g)-B(f, g), \quad f, g \in C_{0}(S) .
$$

The bimeasure $B^{\prime}$ is positive definite, since it follows from the inequality (4) that

$$
B^{\prime}(f, \bar{f})=v\left(|f|^{2}\right)-B(f, \bar{f}) \geqq 0 \quad \text { for all } f \in C_{0}(S) .
$$

Thus the mappings

$$
\begin{aligned}
Q(f, g) & =\overline{B(\bar{f}, g)}, \quad f, g \in C_{0}(S) ; \\
Q^{\prime}(f, g) & =\overline{B^{\prime}(\bar{f}, g)}, \quad f, g \in C_{0}(S) ;
\end{aligned}
$$

are positive definite kernels.

In the following we consider the reproducing kernel Hilbert spaces $H(Q)$ and $H\left(Q^{\prime}\right)$ spanned by $Q$ and respectively by $Q^{\prime}$ and the bounded vector measures $\mu_{Q}$ and $\mu_{Q^{\prime}}$ on $S$ defined as in the formula (3).

We denote

$$
H^{\prime}=H(Q) \oplus H\left(Q^{\prime}\right) \text {. }
$$

The elements of $H^{\prime}$ are ordered pairs $(x, y), x \in H(Q), y \in H\left(Q^{\prime}\right)$; and the inner product of two elements $\left(x_{1}, y_{1}\right),\left(x_{2}, y_{2}\right) \in H^{\prime}$ is

$$
\left(\left(x_{1}, y_{1}\right) \mid\left(x_{2}, y_{2}\right)\right)_{H^{\prime}}=\left(x_{1} \mid x_{2}\right)_{H(Q)}+\left(y_{1} \mid y_{2}\right)_{H\left(Q^{\prime}\right)} \text {. }
$$

We define a linear mapping $j: \mu\left(C_{0}(S)\right) \rightarrow H^{\prime}$ by setting

$$
j(\mu(f))=\left(\mu_{Q}(f), 0\right), \quad f \in C_{0}(S) .
$$

Since $\|\mu(f)\|_{H}=\|j(\mu(f))\|_{H^{\prime}}$ for all $f \in C_{0}(S)$ the linear mapping $j$ can be extended by continuity to an isometric isomorphism $j: \overline{\mathrm{sp}}\{\mu\} \rightarrow j(\overline{\mathrm{sp}}\{\mu\})$. Note that $j(\overline{\mathrm{sp}}\{\mu\})=$ $=H(Q) \oplus\{0\}$. 
To complete the construction of the orthogonally scattered dilation $\left(H^{\prime}, \mu^{\prime}, j\right)$ of $(H, \mu)$ we define a bounded $H^{\prime}$-valued vector measure $\mu^{\prime}$ on $S$ by setting

$$
\mu^{\prime}(f)=\left(\mu_{Q}(f), 0\right)+\left(0, \mu_{Q^{\prime}}(f)\right), \quad f \in C_{0}(S) .
$$

The vector measure $\mu^{\prime}$ is, by Theorem 7 , orthogonally scattered, since for all $f, g \in C_{0}(S)$

$$
\begin{aligned}
\left(\mu^{\prime}(f) \mid \mu^{\prime}(g)\right)_{H^{\prime}} & =\left(\mu_{Q}(f) \mid \mu_{Q}(g)\right)_{H(Q)}+\left(\mu_{Q^{\prime}}(f) \mid \mu_{Q^{\prime}}(g)\right)_{H\left(Q^{\prime}\right)} \\
& =B(f, \bar{g})+B^{\prime}(f, \bar{g})=v(f \bar{g}) .
\end{aligned}
$$

Since $j(\overline{\mathrm{sp}}\{\mu\})=H(Q) \oplus\{0\}$ we get

$$
j \circ \mu(f)=\left(\mu_{Q}(f), 0\right)=P_{j(\overline{\mathrm{sp}}\{\mu\})} \circ \mu^{\prime}(f)
$$

for all $f \in C_{0}(S)$. Hence the triple $\left(H^{\prime}, \mu^{\prime}, j\right)$ is an orthogonally scattered dilation of the pair $(H, \mu)$ satisfying the condition (4).

The theorem is proved.

We are now ready to present the theorem that has been aimed at in this paper. It is a direct consequence of Theorem 4 and Theorem 12.

Theorem 13. Let $H$ be a Hilbert space and let $\mu$ be a bounded H-valued vector measure on $S$. Then the set of orthogonally scattered dilations of $(H, \mu)$ is non-empty. For every 2-majorant $v \in \mathscr{M}_{\mu}^{2}$ there exists an orthogonally scattered dilation $\left(H^{\prime}, \mu^{\prime}, j\right)$ of $(H, \mu)$ such that

$$
\left(\mu^{\prime}(f) \mid \mu^{\prime}(g)\right)=v(f \bar{g}) \quad \text { for all } f, g \in C_{0}(S) .
$$

As an example we present the special case considered by Abreu.

Example 14. Let $\mu$ be a bounded vector measure defined on $S$ with values in a Hilbert space $H$ and let $B$ be the bimeasure defined by $\mu$.

Suppose there exists a bounded Radon measure $v \in \mathscr{M}_{C}^{1}(S \times S)$ such that

$$
B(f, g)=v(f \otimes g) \quad \text { for all } f, g \in C_{0}(S) ;
$$

here $f \otimes g(s, t)=f(s) g(t)$ for all $s, t \in S$.

If there exists a bounded Radon measure $v \in \mathscr{M}_{C}^{1}(S \times S)$ satisfying the condition (6), then it is unique [8; p. 23].

Let $|v|$ be the absolute value of $v$. Define a bounded Radon measure $v_{0}: C_{0}^{\mathrm{Re}}(S) \rightarrow R$ by setting

$$
v_{0}(f)=\frac{1}{2} \int(f \otimes 1+1 \otimes f) d|v|, \quad f \in C_{0}^{\mathrm{Re}}(S)
$$

here again 1 stands for the constant function $g(s)=1, s \in S$.

Abreu has shown in the proof of the main theorem in [1] that

$$
\|\mu(f)\|^{2} \leqq v_{0}\left(|f|^{2}\right) \quad \text { for all } f \in C_{0}(S) .
$$


Thus, using our terminology, the bounded positive Radon measure $\tilde{v}_{0}, \tilde{v}_{0}(f)=$ $=v_{0}(\operatorname{Re} f)+i v_{0}(\operatorname{Im} f), f \in C_{0}(S)$, is a 2-majorant of $\mu$.

Acknowledgement. This work has been supported by the Magnus Ehrnrooth Foundation.

\title{
References
}

[1] Abreu, J. L.: A note on harmonizable and stationary sequences. - Bol. Soc. Mat. Mexicana $15: 2,1970,48-51$.

[2] Abreu, J. L.: $H$-valued generalized functions and orthogonally scattered measures. - Centro de Investigácion en Matemáticas Aplicadas y en Sistemas, Universidad Nacional Autónoma de México. Comunicaciones tecnicas, Serie B: Investigación 6:81, 1975.

[3] Aronszajn, N.: Theory of reproducing kernels. - Trans. Amer. Math. Soc. 68, 1950, 337 404.

[4] BourbaKI, N.: Éléments de mathématique. XXI. Intégration. Chapitre 5. 2ième éd. - Actualités Sci. Indust. 1244, Hermann et $\mathrm{C}^{\text {ie }}$, Paris, 1967.

[5] Grothendieck, A.: Résumé de la théorie métrique des produits tensoriels topologiques. - Bol. Soc. Mat. São Paulo 8, 1956, 1-79.

[6] Masani, P.: Orthogonally scattered measures. - Advances in Math. 2, 1968, 61-117.

[7] Morse, M., and W. TRAnsue: $C$-bimeasures $\Lambda$ and their integral extensions. - Ann. of Math. (2) $64,1956,480-504$.

[8] Niemi, H.: Stochastic processes as Fourier transforms of stochastic measures. - Ann. Acad. Sci. Fenn. Ser. A I 591, 1975, 1-47.

[9] NIEMI, H.: On the support of a bimeasure and orthogonally scattered vector measures. - Ibid. 1, 1975, 249-275.

[10] PIETSCH, A.: p-majorisierbare vektorwertige Maße. - Wiss. Z. Friedrich-Schiller-Univ. Jena/Thüringen Math.-Natur. Reihe 18, 1969, 243-247.

[11] Rogge, R.: Maße mit Werten in einem Hilbertraum. - Ibid. 18, 1969, 253-257.

[12] Thomas, E.: L'intégration par rapport à une mesure de Radon vectorielle. - Ann. Inst. Fourier (Grenoble) 20:2, 1970, 55-191.

\author{
University of Helsinki \\ Department of Mathematics \\ SF-00100 Helsinki 10
}

Finland

Received 15 September 1975 